\title{
CLINICAL MEDICINE
}

\section{ОПЫТ ЛЕЧЕНИЯ ГРЫЖ ПИЩЕВОДНОГО ОТВЕРСТИЯ ДИАФРАГМЫ}

\author{
П. Н. Зубарев ${ }^{1}$, И. Е. Онницев ${ }^{1}$, А. В. Хохлов ${ }^{1}$, В. М. Трофимов ${ }^{1}$, В. Л. Белевич ${ }^{1}$, А. Л. Мужаровский ${ }^{1}$ \\ ${ }^{1}$ ФГБВоУ В0 «Военно-медицинская академия имени С. М. Кирова» МО РФ, г. Санкт-Петербург, Россия
}

\section{EXPERIENCE IN TREATMENT OF DIAPHRAGM HERNIAS}

\author{
P. N. Zubarev' ${ }^{1}$ I. E. Onnitsev' ${ }^{1}$, A. V. Khokhlov' ${ }^{1}$, V. M. Trofimov' ${ }^{1}$, V. L. Belevich' ${ }^{1}$, A. L. Muzharovskiy ${ }^{1}$ \\ ${ }^{1}$ S. M. Kirov Military Medical Academy of the Russian Defense Ministry, Saint Petersburg, Russia
}

\begin{abstract}
Резюме. Грыжи пищеводного отверстия диафрагмы являются распространенной патологией, негативно влияющей на качество жизни пациентов и требующей в некоторых случаях хирургического лечения. Она обладает прогредиентным течением, что приводит к увеличению степени тяжести клинических проявлений с возрастом пациента. В статье приведен опыт хирургического лечения грыжи пищеводного отверстия диафрагмы на базе клиники общей хирургии ВМедА. Цель исследования - определить эффективность хирургического лечения грыжи пищеводного отверстия диафрагмы у разной категории больных. Общее количество пролеченных больных составило 216 человек. Все пациенты были прооперированы. 34 больным проведена симультанная лапароскопическая холецистэктомия. При операции использовался лапароскопический доступ. Ближайшие отличные и хорошие результаты лечения по клиническим данным имели место в $92 \%$ случаев, на долю удовлетворительных и неудовлетворительных результатов пришлось соответственно 5,4 и 2,7\% случаев. По результатам инструментального обследования показатели отличных и хороших результатов составили соответственно 40,6 и 54\%; удовлетворительных и неудовлетворительных 2,7 и 2,7\%. Внедрение лапароскопических техник в хирургию позволило снизить число ранних и поздних послеоперационных осложнений при лечении грыжи пищеводного отверстия диафрагмы. В рекомендациях Американской ассоциации эндоскопических хирургов по оперативному лечению гастроэзофагеальной рефлюксной болезни показан положительный эффект лапароскопических фундопликаций у 85-93\% пациентов, медикаментозная терапия у которых оказалась неэффективной. В целом, результаты ближайших и отдаленных исходов хирургического лечения грыжи пищеводного отверстия диафрагмы в клинике общей хирургии ВМедА продемонстрировали схожие результаты эффективности (8 рис., библ.: 16 ист.).
\end{abstract}

Ключевые слова: грыжи пищеводного отверстия диафрагмы, лечение.

Статья поступила в редакцию 30.09.2020 г.

\section{ВВЕДЕНИЕ}

Грыжи пищеводного отверстия диафрагмы (ГПОД) являются одной их актуальнейших проблем современной хирургии и гастроэнтерологии [1]. Частота ГПОД в структуре патологии желудочнокишечного тракта колеблется от 3 до 33\%, занимая 3-е место после желчнокаменной болезни, язвенной болезни желудка и 12-перстной кишки [3]. ГПОД обладает прогредиентным течением, что приводит к увеличению степени тяжести клинических проявлений с возрастом пациента [4]. Клиническое
Summary. Hernias of the diaphragm are a common pathology that negatively affects the quality of life of patients and in some cases requires high-quality surgical correction. This pathology has a progressive course, which leads to an increase in the severity of clinical manifestations with the age of the patient. The article presents the experience of surgical treatment of hernias of the diaphragm on the basis of general surgery clinics of the military medical academy. The aim of the study is to determine the effectiveness of surgical treatment of hernias of the diaphragm in different categories of patients. The total number of treated patients was 216. All patients were operated on. Thirty-four patients underwent a sympultal laparoscopic cholecystectomy. The operation used laparoscopic access. The closest excellent and good results of clinical treatment occurred in $92 \%$ of cases, satisfactory and unsatisfactory results accounted for 5.4 and $2.7 \%$, respectively. According to the results of the instrumental survey, 40.6 and $54 \%$, respectively, achieved excellent and good results; Satisfactory and unsatisfactory -2.7 and $2.7 \%$. The introduction of laparoscopic techniques in surgery has reduced the number of early and late post-operative complications in the treatment of hernias of the diaphragm. The recommendations of the American Association of Endoscopic Surgeons for the operational treatment of gastroesophageal reflux disease shows the positive effect of laparoscopic fundoplications in $85-93 \%$ of patients whose medicinal therapy has proved ineffective. In general, the results of the immediate and long-term outcomes of the surgical treatment of hernias of the diaphragm at the General Surgery Clinic of Military Medical Academy showed similar results (8 figs, bibliography: 16 refs).

Key words: hernias of the diaphragm, treatment.

Article received 30.09.2020

течение ГПОД сопровождается значительным снижением качества жизни больных и не поддается терапевтическому лечению. В генезе ГПОД ведущую роль отводят наследственной предрасположенности и врожденной слабости соединительной ткани $[5,6]$. При данной патологии происходят структурная деградация соединительно-тканных волокон пищеводно-диафрагмальной мембраны, ослабление связочного аппарата желудка или других органов $[7,8]$. Предрасполагающими факторами являются ожирение, неправильная осанка, хроническое 
повышение внутрибрюшного давления (упорный кашель, запор) [9]. В настоящее время выделяют несколько типов ГПОД [10]: I тип (аксиальные, скользящие), при котором происходит осевое смещение пищеводно-желудочного перехода, дна или тела желудка в средостение через расширенное пищеводное диафрагмальное отверстие и возвращение в брюшную полость в случае перемены положения тела; II тип (параэзофагеальные), при котором пищеводно-желудочный переход располагается в брюшной полости, а часть желудка перемещается в средостение; III тип (смешанные), при котором наблюдаются анатомические изменения аксиальных и параэзофагеальных грыж; IV тип, при котором в средостение смещаются другие органы брюшной полости, такие как сальник, тонкая и толстая кишка, поджелудочная железа, связки печени.

Как правило, у половины пациентов грыжа пищеводного отдела диафрагмы протекает либо бессимптомно, либо с минимальным набором клинических проявлений, многие из которых не являются строго специфичными для данного заболевания. Поэтому чаще всего ГПОД выявляются случайно в ходе диагностических исследований, предпринимаемых по поводу других заболеваний [11]. Большинством исследователей подчеркивается, что с момента появления каких-либо симптомов до момента обращения к врачу и установки верного диагноза, как правило, проходит длительное время. Чаще всего пациенты с ГПОД наблюдаются у гастроэнтеролога по поводу рефлюкс-эзофагита. Лечение начинается с поведенческой и консервативной терапии - уменьшения физической нагрузки, рационального режима питания и диеты, фармакотерапии ингибиторами протонной помпы, Н2-блокаторами гистаминовых рецепторов, прокинетиками. Исход консервативного лечения зависит от многих факторов - возраста пациента, наличия сопутствующей патологии, степени тяжести патологического процесса и т. п. Поэтому эффективность консервативного лечения, по данным литературы, находится в диапазоне 23-75\% [12]. При отсутствии эффективности консервативной терапии пациентам с ГПОД рекомендуется хирургическое лечение. Наиболее распространённым методом оператив- ного лечения ГПОД в настоящее время является лапароскопическая задняя фундопликация по Ниссену с крурорафией $[2,14]$. Данный метод считается наиболее адекватным способом восстановления барьерной функции желудочно-пищеводного перехода. Так как часть ГПОД являются случайными диагностическими находками, протекающими бессимптомно либо с минимальными клиническими проявлениями, а подавляющим большинством пациентов с этой патологией являются люди среднего и пожилого возраста, имеющие сопутствующую соматическую патологию, существуют определенные сложности в определении показаний к хирургическому лечению ГПОД [15]. Показанием к оперативному лечению аксиальных грыж пищеводного отверстия диафрагмы является неэффективность консервативной терапии гастроэзофагальной рефлюксной болезни. Хирургические вмешательства при бессимптомных аксиальных грыжах не показаны [16]. Показанием к хирургическому лечению параэзофагеальных и смешанных ГПОД является наличие их клинических проявлений. Развитие острых осложнений - ущемление ГПОД, непроходимость при параэзофагеальных и смешанных ГПОД - является показанием к неотложной операции. При ГПОД IV типа хирургическое лечение показано при наличии клинических проявлений или выявлении в грыжевом выпячивании петель тонкой или толстой кишки [13]. Эффективность хирургического лечения ГПОД, по разным источникам, достигает 75-95\% [9, 13, 15]. В клинике общей хирургии ВМедА за последние 10 лет накоплен большой опыт хирургической коррекции ГПОД, результаты которого могут представлять интерес для практикующих хирургов.

\section{ЦЕЛЬ ИССЛЕДОВАНИЯ}

Определить эффективность хирургического лечения ГПОД у разной категории больных.

\section{МАТЕРИАЛЫ И МЕТОДЫ}

Общее количество пролеченных больных составило 216 человек, из них 43 мужчины и 173 женщины. Распределение пациентов по возрасту (по классификации ВО3) представлено на рис. 1.

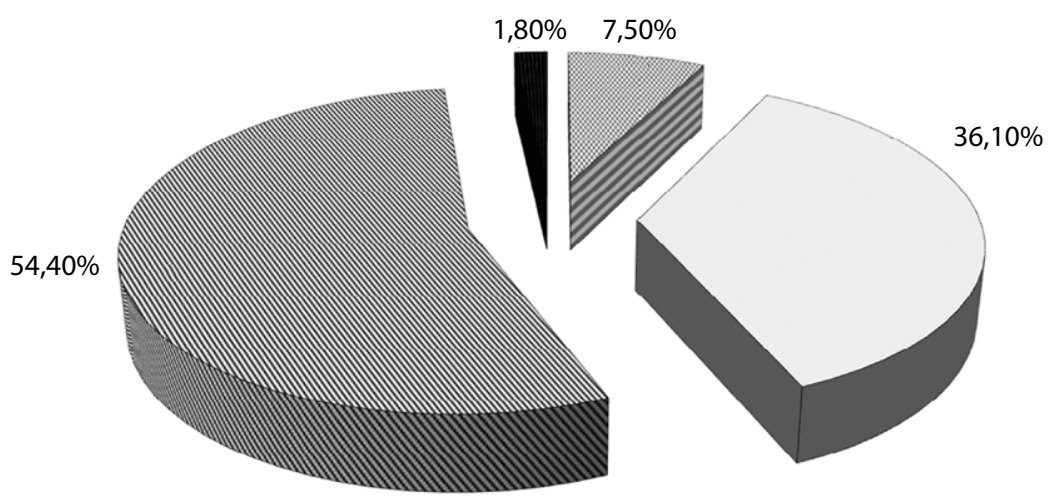

ФМолодой возраст

口Средний возраст

凶Пожилой возраст

Старческий возраст

Рис. 1. Структура больных по возрасту 


\section{CLINICAL MEDICINE}

Структура типов ГПОД у госпитализированных пациентов представлена на рис. 2.

Показаниями к оперативному лечению были: неэффективность консервативной терапии ГПОД $(34,5 \%)$, рецидивирующий рефлюкс-эзофагит $(30,4 \%)$, степень ГПОД по данным дополнительных методов исследования (52,5\%). Все пациенты были прооперированы, 34 больным проведена симультанная лапароскопическая холецистэктомия. При операции использовался лапароскопический доступ. Размеры пищеводного отверстия диафрагмы у прооперированных больных представлены на рис. 3.

Содержимое грыжевого мешка было представлено у 53,4\% кардиальным отделом желудка, у $44,1 \%$ - дном и телом желудка, у 2,5\% содержало весь желудок, петли тонкой или толстой кишки. У всех больных коррекция ГПОД проводилась с формированием циркулярной фундопликационной манжеты, размер которой составил $3,4 \pm 0,4$ см.

Ближайшие результаты лечения (через 6 мес.) оценены у 97 пациентов. Для оценки результатов лечения использовались клинические и инструментальные данные согласно критериям H. Wykypiel с соавторами (2005).

Оказалось, что ближайшие отличные и хорошие результаты лечения по клиническим данным имели место в 92\% случаев, на долю удовлетворительных и неудовлетворительных результатов пришлось соответственно 5,4 и 2,7\% случаев. По результатам инструментального обследования показатели отличных и хороших результатов составили соответственно 40,6 и 54\%; удовлетворительных и неудовлетворительных - 2,7 и 2,7\% (рис. 2).

Отдаленные результаты в сроки от 24 до 36 мес. изучены у 51 пациента с использованием тех же критериев. По клиническим данным (рис. 3) отличные и хорошие результаты выявлены в 57,2 и 33,3\% случаев; удовлетворительные - в 9,5\% случаев.

Интраоперационные осложнения выявлены у 8 (3,4\%) больных. В шести наблюдениях диагностирован правосторонний пневмоторакс, который был связан с повреждением медиастинального листка париетальной плевры. В двух случаях

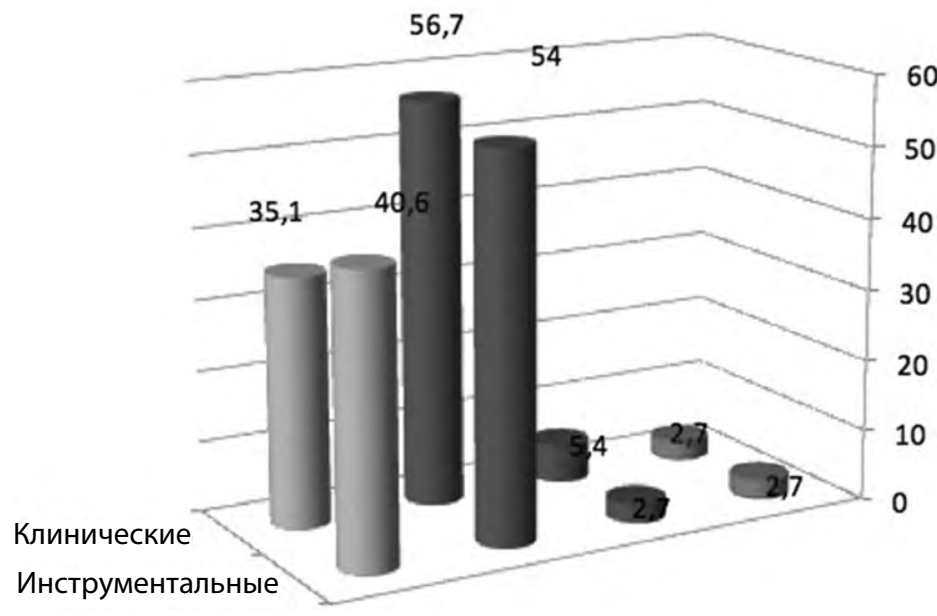

— Отличные

- Хорошие

- Удовлетворительные

च Неудовлетворительные

प

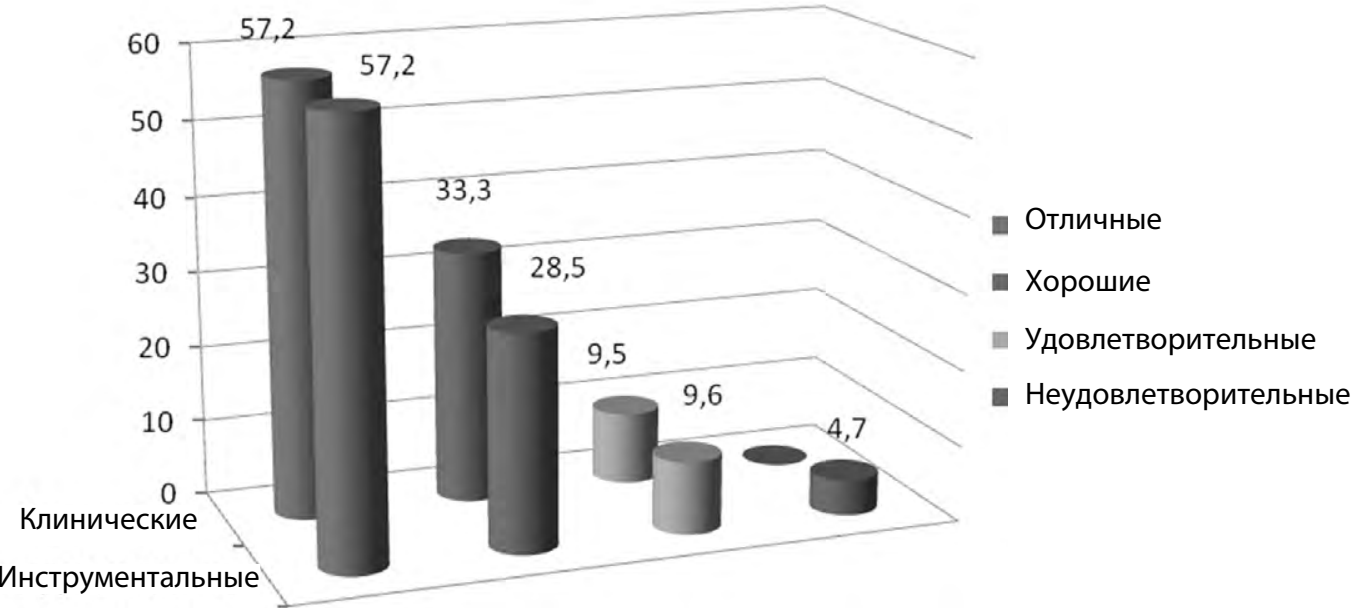

Рис. 3. Отдаленные результаты лечения по данным клинического и инструментального обследования 
отмечена эмфизема области грудной клетки, шеи, лица. Во всех случаях интраоперационные осложнения не повлияли на выполнение запланированного объема операции.

Таким образом, при неосложненном упорном течении рефлюксассоциированного эзофагита на фоне наличия ГПОД после курса консервативной терапии, направленной на ликвидацию воспаления слизистой пищевода, показано лапароскопическое устранение грыжи с выполнением антирефлюксной операции.

При осложненном течении заболевания, по нашему мнению, следует прибегать к двухэтапному эндовидеохирургическому лечению. На первом этапе с помощью гибкой внутрипросветной эндовидеоэзофагоскопии следует устранить органические и функционально значимые осложнения, на втором этапом (по показаниям) - выполнить устранение ГПОД с антирефлюксной операцией эндовидеохирургическим способом. Операцией выбора, по нашему мнению, следует считать лапароскопическую крурорафию с фундопликацией по Ниссен-Розетти.

Мы решили представить один клинический случай неблагоприятного течения ГПОД. Больная Д., 79 лет, доставлена бригадой скорой медицинской помощи 02.01.2020 г. в приемное отделение ВМедА с жалобами на ноющие, распирающие боли, чувство тяжести в эпигастрии иррадиирующие в область сердца при употреблении пищи, изжогу, отрыжку тухлым воздухом, тошноту, неоднократную рвоту. Из анамнеза заболевания известно, что тошнота беспокоит больную в течение последних 3 нед, рвота появилась внезапно. Отмечает быстрое чувство насыщения после приема небольшого количества пищи. После приема спазмолитиков и самостоятельно вызванной рвоты боль стихает. Приступ боли длится примерно 1-1,5 ч, возникает 1-3 раза в день. Развитие и течение болезни: больная отмечает, что боли в эпигастрии беспокоят 10 лет. С декабря 2019 г. беспокоят вышеописанные жалобы. В октябре 2019 г. проходила лечение в хирургическом стационаре по поводу анемии. При обследовании обнаружены грыжа пищеводного отверстия диафрагмы IV степени, гипохромная анемия (рис. 4). Предложено плановое оперативное лечение ГПОД. Сопутствующая патология: атеросклероз аорты и ее ветвей; гипертоническая болезнь 2-й ст. (риск ССО-4); недостаточность кровообращения степень 2А функциональный класс ишемической болезни сердца (НК-2А фк. ИБС); хронический гастрит; хронический некалькулезный холецистит; хронический панкреатит; аденома надпочечников; парапельвикальная киста левой почки; мочекаменная болезнь (МКБ), камни и микролиты почек; дивертикулез нисходящей ободочной и сигмовидной кишки; антелистез $\mathrm{L}_{4}$ позвонка; неполная правосторонняя сакрализация $\mathrm{L}_{5}$; язвенная болезнь две-

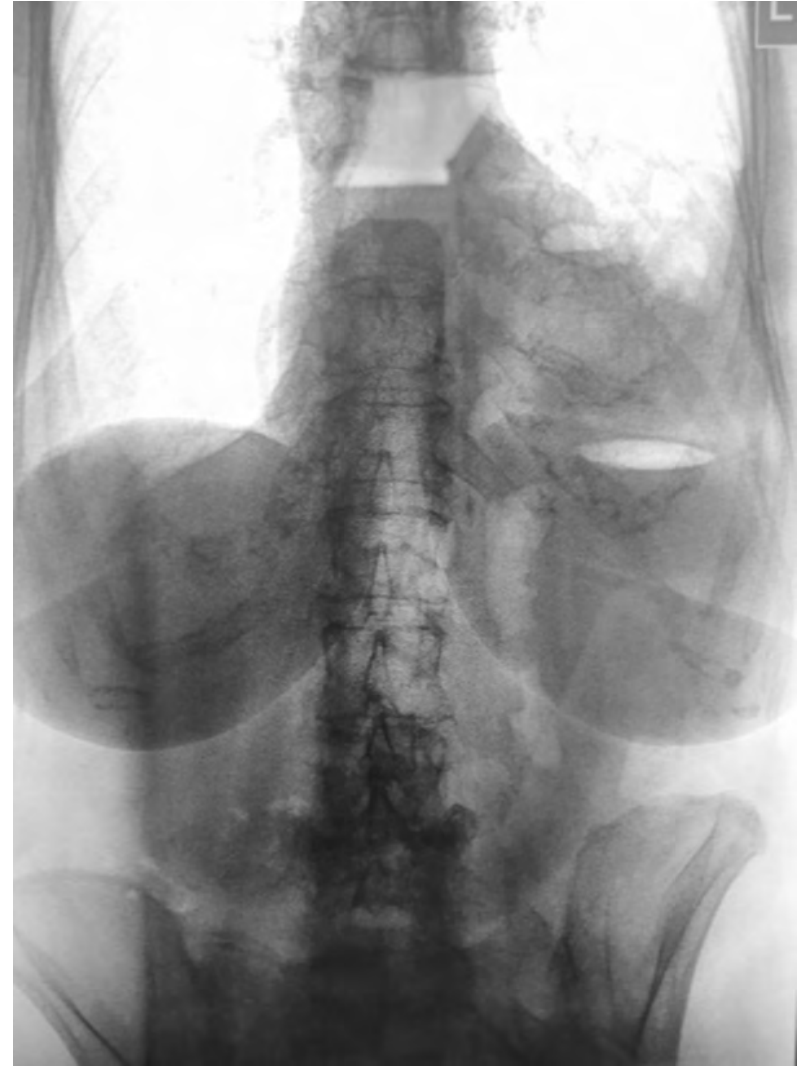

Рис. 4. Параэзофагеальная ГПОД (грудной желудок). Сдавление двенадцатиперстной кишки

надцатиперстной кишки, ремиссия; хронический гастродуоденит.

При поступлении состояние больной средней степени тяжести. Рост - 156 см, вес - 62 кг. Кожные покровы бледноваты, чистые. Дыхание везикулярное, хрипов нет. Перкуторный звук ясный легочной. Тоны сердца ритмичные, ясные. АД 110/70 мм рт. ст., пульс 64 уд./мин. Язык влажный, живот мягкий, безболезненный. Объемные образования в брюшной полости не пальпируются. Перистальтика выслушивается. Симптом Щеткина-Блюмберга отрицательный. Симптом сотрясения с обеих сторон отрицателен. Лабораторные данные. Развернутый анализа крови: лейкоциты - 14,0 × 109/л.; палочкодерные (п/я) - 6; сегментоядерные (с/я) - 75; лимфоциты - 10; моноциты - 9; гемоглобин (Нb) 113 г/л; СОЭ - 43 мм/ч; анизоцитоз +. Результаты биохимических показателей крови: К+ 3,07; мочевина 20,0 ммол/л; креатинин - 155 ммол/л. Общий анализ мочи: кол-во - 50 мл; цвет - мутная; уд. вес - 1007; эпит. плоский - 9-11 в п/3; лейкоциты - 15-20 в п/3; эритроциты - 4-6 в п/3; слизь +; оксалаты +. Инструментальные методы исследования. Ультразвуковое исследование органов брюшной полости от 02.01.2020 г.: УЗ-признаки диффузных изменений печени по типу фиброза; полипы желчного пузыря; диффузные изменения поджелудочной железы; МКБ, камни и микролиты обеих почек; гастростаз. Видеогастродуоденоскопия 


\section{CLINICAL MEDICINE}

от 05.01.2020 г.: кардия расположена на 34 см от резцов, определяется выраженная деформация просвета за счет смещенного желудка на 3/4 выше хиатального отверстия. В просвете большое количество застойного содержимого (более 1,0 л) и его отсутствие в антральном отделе. Выполнено заведение двухпросветного питательного зонда диаметром $12 \mathrm{Fr}$ до уровня связки Трейца. Тотальная параэзофагеальная грыжа. Эритематозная гастропатия. Деформация луковицы двенадцатиперстной кишки. Фунуция внешнего дыхания (ФВД) от 10.01.2020: ФВД сохранена. Жизненная емкость легких - нижняя граница нормы. Рентгенография желудка: нижняя 1/3 пищевода образует изгиб в грудной полости. Весь желудок, газовый пузырь расположен в грудной полости. Желудок заполнен контрастом по малой кривизне на задне-боковой стенке втяжения. Эвакуации контраста из желудка нет.

Компьютерная томография органов грудной клетки, органов брюшной полости от 02.01.2020 г. (рис. 5): гигантская грыжа пищеводного отверстия диафрагмы с наличием компрессионных изменений в прилегающей легочной ткани; жидкость в полости перекарда; коронаросклероз; деформация желчного пузыря; повышение плотностных показателей желчи; КТ-картина образования обоих надпочечников; кистозное образование в синусе левой почки - парапельвикальная киста; дивертикулез нисходящей ободочной и сигмовидной кишки; формирующаяся пупочная грыжа; атеросклероз аорты и ее ветвей; антелистез $\mathrm{L}_{4}$ позвонка; неполная правосторонняя сакрализация $\mathrm{L}_{5}$.

В течение сут на фоне инфузионной, спазмолитической терапии существенной положительной динамики не отмечалось, продолжалась рвота до 8 раз в сут, сопровождающаяся выраженным астеническим синдромом, сохранялись боли в левом подреберье и эпигастральной области. В связи с со-

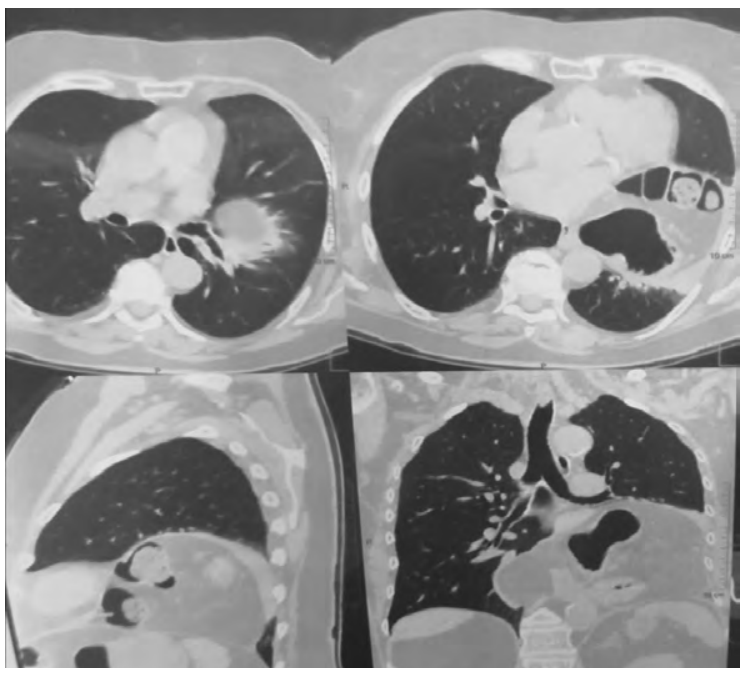

Рис. 5. КТ-признаки гигантской грыжи пищеводного отверстия диафрагмы храняющейся выраженной рвотой и симптомами дуоденальной непроходимости, а также с целью подготовки пациентки к оперативному лечению под эндоскопическим контролем установлен двухпросветный питательный зонд в просвет желудка и двенадцатиперстной кишки (рис. 6).

После компенсации водно-электролитных нарушений, стабилизации пациентки была выполнена плановая операция 11.01.20 г.: пластика диафрагмальной грыжи, задняя и передняя диафрагмокрурорафия, фундопликация по Ниссену. При ревизии обнаружено, что желудок полностью смещен в средостение через пищеводное отверстие диафрагмы и фиксирован там рубцово-спаечным процессом. В грыжевом мешке также располагаются поперечно-ободочная кишка и петля тонкой кишки. Ультразвуковыми ножницами рассечены спайки и рубцы. Поперечно-ободочная кишка, желудок низведены в брюшную полость. С помощью ультразвукового диссектора пересечены короткие желудочные сосуды, мобилизован свод пищевода, выделена его нижняя треть. Пищеводное отверстие дифрагмы расширено до 10 см (рис. 7).

Ножки диафрагмы сшиты позади и спереди пищевода до нормального физиологического размера (рис. 8А). Наложены узловые швы на дно желудка, переднюю стенку пищевода с формированием муфты (рис. 8Б).

Установлен дренаж в поддиафрагмальное пространство слева и полость грыжи.

Пациентка выписана на 7-е сут.

Таким образом, рассмотренный нами клинический случай представляетопределенный практический интерес и как редкая возможность наблюдать ГПОД IV степени с признаками декомпенсированной дуоденальной непроходимости, так и как демонстрация возможного возникновения определенных трудностей при проведении дифференциальной

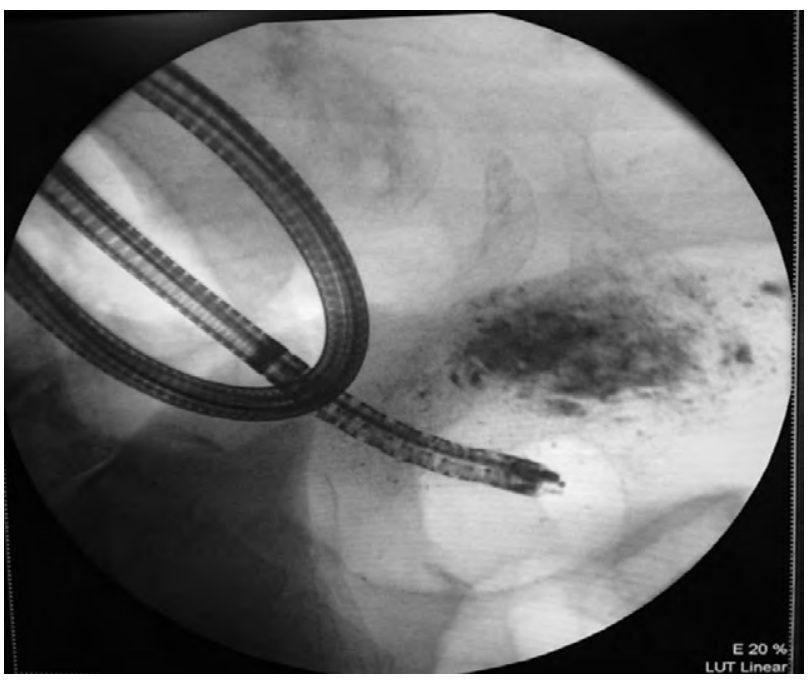

Рис. 6. Установка зонда в просвет двенадцатиперстной кишки 


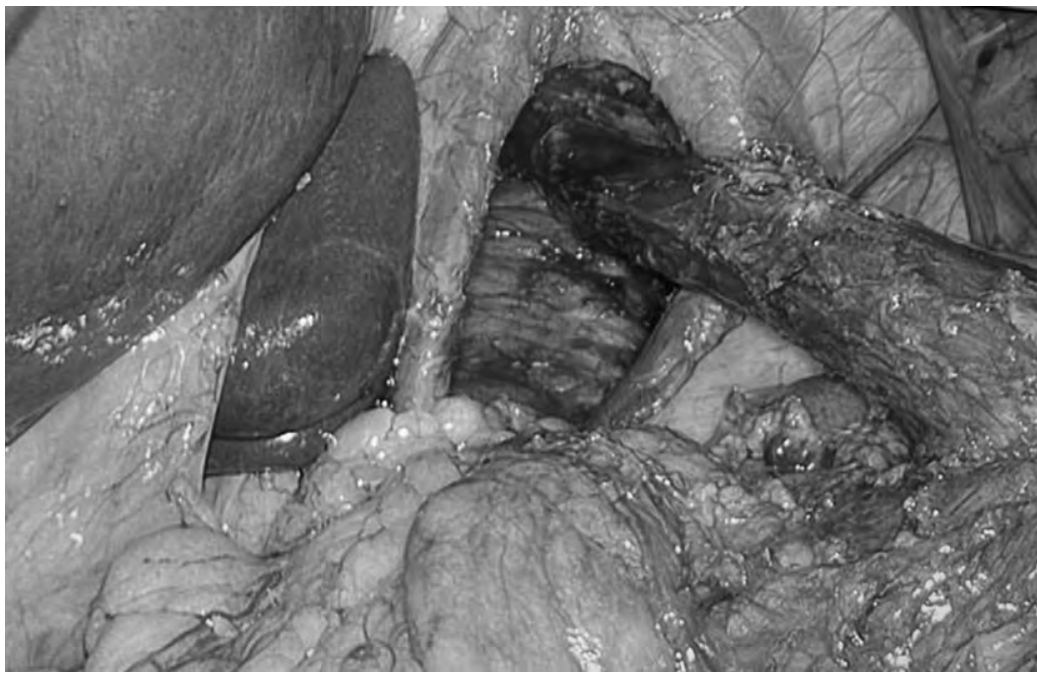

Рис. 7. Мобилизованный пищевод и ножки диафрагмы

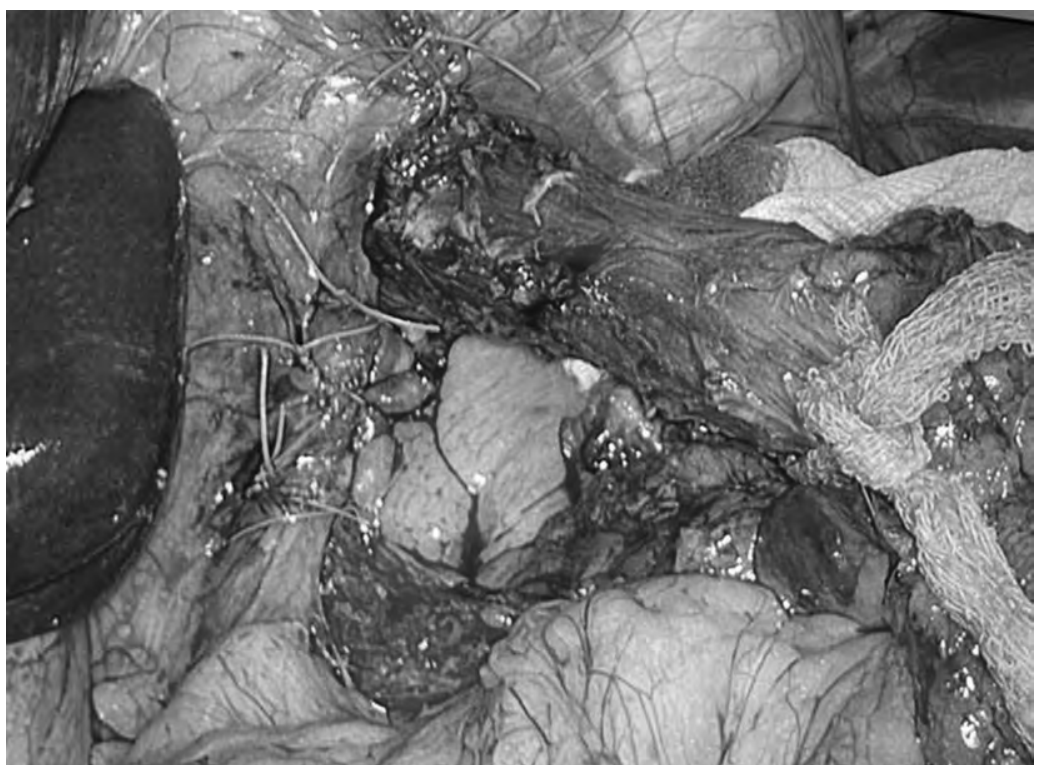

A

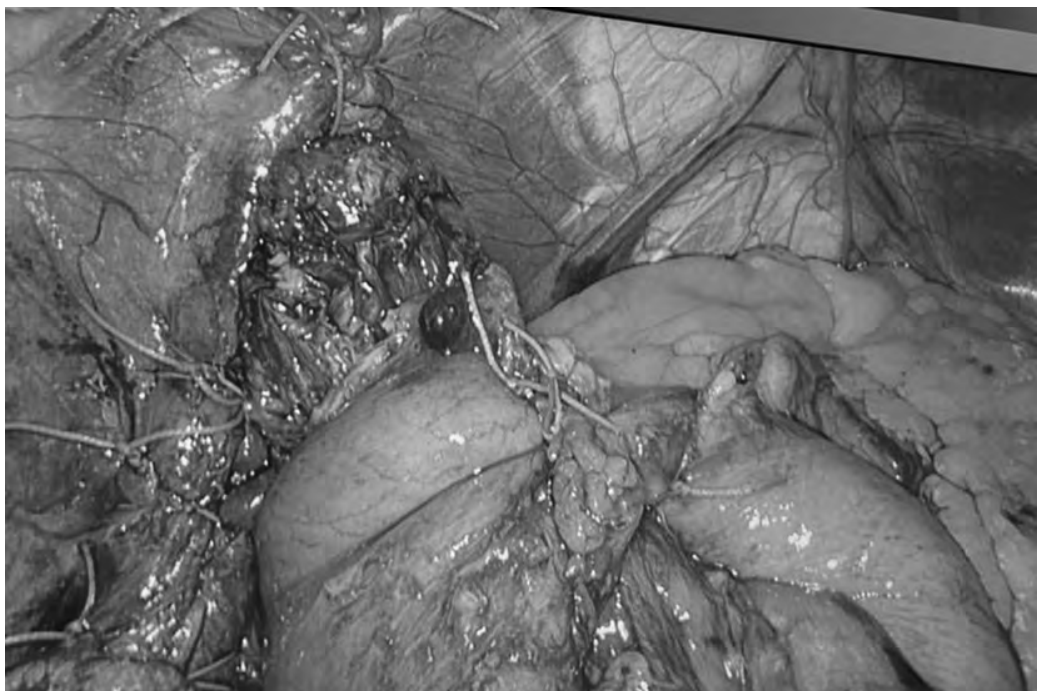

Б

Рис. 8. А - передняя и задняя крурорафия; Б - фундопликация по Ниссен-Розетти 
диагностики, подготовки пациента и выполнении операции лапароскопическим доступом.

\section{ЗАКЛЮЧЕНИЕ}

ГПОД - это заболевание, в генезе которого играют роль множество факторов. Полученные результаты подтверждают данные литературы, свидетельствующие, что ГПОД чаще проявляется у женщин, чем у мужчин. Считается, что основными клиническими проявлениями ГПОД являются пищеводные симптомы, обусловленные течением неэрозивного и эрозивного рефлюкс-эзофагита: изжога, ретростернальные боли, дисфагия, отрыжка и регургитация. Проведенное исследование подтвердило преобладание пищеводных симптомов у больных с ГПОД, которые выявлялись у 95\% пациентов. Существующие в настоящее время эффективные препараты (антацидные, антисекреторные и прокинетические) для лечения гастроэзофагеальной рефлюксной болезни (ГЭРБ), ведущего клинического проявления ГПОД, снизили количество показаний к хирургиче-

\section{УВЕДОМЛЕНИЕ}

Авторы внесли равный вклад в данную работу и сообщают об отсутствии какого-либо конфликта интересов.

\section{СПИСОК ЛИТЕРАТУРЫ / REFERENCES}

1. Clifton M. S., Wulkan M. L. Congenital Diaphragmatic Hernia and Diaphragmatic Eventration. Clin. Perinatol. 2017; 44 (4): 773-9.

2. Tyson A. F., Sola R. Jr., Arnold M. R., Cosper G. H., Schulman A. M. Thoracoscopic Versus Open Congenital Diaphragmatic Hernia Repair: Single Tertiary Center Review. J. Laparoendosc. Adv. Surg. Tech. A. 2017; 27 (11): 1209-16.

3. Zyabreva I. A., Dzhulay T. E. Esophageal hernia of the diaphragm: controversial, unresolved and promising aspects of the problem (literature review). Upper Volga Medical Journal. 2015; 4: 24-8. Russian (Зябрева И. А., Джулай T. Е. Грыжи пищеводного отверстия диафрагмы: спорные, нерешенные и перспективные аспекты проблемы (обзор литературы). Верхневолжский медицинский журнал. 2015; 4: 24-8).

4. Tumarenko A. V., Skvortsov V. V. Hernia of the diaphragm in the practice of a therapist. Krymskiy terapevticheskiy zhurnal. 2015; 4: 80-4. Russian (Тумаренко А. В., Сквориов В. В. Грыжа пищеводного отверстия диафрагмы в практике терапевта. Крымский терапевтический журнал. 2015; 4: 80-4).

5. Fetishcheva L. E., Zakharov I. S., Ushakova G. A., Moses V. G., Demyanova T. N., Vasyutinskaya Yu. V., Petrich L. N. Interstitial pregnancy - diagnostic difficulties. Mat' i ditya v Kuzbasse. 2017; 2(69): 55-58. Russian (Фетищева Л. Е., Захаров И. С., Ушакова Г. А., Мозес В. Г., Демьянова Т. Н., Васютинская Ю. В., Петрич Л. Н. Интерстициальная беременность - трудности диагностики. Мать и дитя в Кузбассе. 2017; 2 (69): 55-8).

6. Inoue M., Uchida K., Otake K., Nagano Y., Mori K., Hashimoto K., Matsushita K., Koike Y., Uemura A., Kusunoki M. Thoracoscopic repair of congenital diaphragmatic hernia with countermeasures against reported complications for safe скому лечению данной патологии. В своей практике мы тоже придерживаемся данной стратегии, оперируя пациентов в том случае, когда консервативные методы лечения грыж неэффективны. Пионером хирургической коррекции ГПОД считается Р. Ниссен, который в 1936 г. первым предложил и выполнил операцию фундопликации, которая до внедрения эндоскопической техники являлась «золотым стандартом» лечения. Внедрение лапароскопических техник в хирургии позволило снизить число ранних и поздних послеоперационных осложнений при лечении ГПОД. В рекомендациях Американской ассоциации эндоскопических хирургов по оперативному лечению ГЭРБ показан положительный эффект лапароскопических фундопликаций у 85-93\% пациентов, в отношении которых медикаментозная терапия х оказалась неэффективной [16]. В целом, результаты ближайших и отдаленных исходов хирургического лечения ГПОД в клинике общей хирургии ВМедА продемонстрировали схожие по эффективности результаты.

\section{ACKNOWLEDGMENT}

Authors contributed equally into this work and declare no conflict of interest.

outcomes comparable to laparotomy. Surg. Endosc. 2016; 30 (3): 1014-9.

7. Herman A., Maiti A., Cherian S. V., Estrada-Y-Martin R. M. Heimlich Maneuver-Induced Diaphragmatic Rupture and Hiatal Hernia. Am. J. Med. Sci. 2018; 355 (4): e13.

8. Sigal E. I., Burmistrov M. V. Benign diseases of the esophagus. Tikhookeanskiy meditsinskiy zhurnal. 2009; 2: 40-7. Russian (Сигал Е. И., Бурмистров М. В. Доброкачественные заболевания пищевода. Тихоокеанский медицинский журнал. 2009; 2: 40-7).

9. Grintsov A. G., Sovpel' O. V., Shapovalov Yu. A., Mate V. V. Functional results of laparoscopic surgery for hiatal hernia. Vestnik neotlozhnoy i vosstanovitel'noy meditsiny. 2012; 2: 242-5. Russian (Гринцов А. Г., Совпель О. В., Шаповалов Ю. А., Мате В. В. Функциональные результаты лапароскопических операций при грыжах пищеводного отверстия диафрагмы. Вестник неотложной и восстановительной медицины. 2012; 13 (2): 242-5).

10. Shwaartz C., Duggan E., Lee D. S., Divino C. M., Chin E. H. Diaphragmatic eventration presenting as a recurrent diaphragmatic hernia. Ann. R. Coll Surg. Engl. 2017; 99 (7): e196-9.

11. Akhmatov A. M., Tarbaev I. S., Vasilevsky D. I. Surgical treatment of hiatal hernia of type II-IV diaphragm. Vestnik khirurgii. 2019; 1: 90-2. Russian (Ахматов А. М., Тарбаев И. С., Василевский Д. И. Хирургическое лечение грыж пищеводного отверстия диафрагмы II-IV типов. Вестник хирургии. 2019; 178 (1): 90-2).

12. Pham D. V., Protyniak B., Binenbaum S. J., Squillaro A., Borao F. J. Simultaneous laparoscopic paraesophageal hernia repair and sleeve gastrectomy in the morbidly obese. Surg. Obes. Relat. Dis. 2014; 10 (2): 257-61. 
13. Dallemagne B., Quero G., Lapergola A., Guerriero L., Fiorillo C., Perretta S. Treatment of giant paraesophageal hernia: pro laparoscopic approach. Hernia. 2018; 22 (6): 909-19.

14. Siegal S. R., Dolan J. P., Hunter J. G. Modern diagnosis and treatment of hiatal hernias. Langenbecks. Arch. Surg. 2017; 402 (8): 1145-51.

\section{СВЕДЕНИЯ ОБ АВТОРАХ}

Зубарев Петр Николаевич - докт. мед. наук, доцент, профессор кафедры общей хирургии, ФГБВОУ ВО «Военномедицинская академия имени С. М. Кирова» МО РФ, 194044, Россия, г. Санкт-Петербург, ул. Академика Лебедева, д. 6

Хохлов Алексей Валентинович - докт. мед. наук, профессор, профессор кафедры общей хирургии, ФГБВОУ ВО «Военно-медицинская академия имени С. М. Кирова» МО РФ, 194044, Россия, г. Санкт-Петербург, ул. Академика Лебедева, д. 6

Онницев Игорь Евгеньевич - докт. мед. наук, старший преподаватель кафедры общей хирургии, ФГБВОУ ВО «Военно-медицинская академия имени С. М. Кирова» МО РФ, 194044, Россия, г. Санкт-Петербург, ул. Академика Лебедева, д. 6

Трофимов Владислав Михайлович - докт. мед. наук, доцент, доцент кафедры общей хирургии, ФГБВОУ ВО «Военно-медицинская академия имени С. М. Кирова» МО РФ, 194044, Россия, г. Санкт-Петербург, ул. Академика Лебедева, д. 6

Белевич Валерий Леонтьевич - докт. мед. наук, старший преподаватель кафедры общей хирургии, ФГБВОУ ВО «Военно-медицинская академия имени С. М. Кирова» МО РФ 194044, Россия, г. Санкт-Петербург, ул. Академика Лебедева, д. 6

Мужаровский Антон Леонидович - канд. мед. наук, врач-хирург клиники общей хирургии, ФГБВОУ ВО «Военномедицинская академия имени С. М. Кирова» МО РФ, 194044, Россия, г. Санкт-Петербург, ул. Академика Лебедева, д. 6
15. Chan E. G., Sarkaria I. S., Luketich J. D., Levy R. Laparoscopic Approach to Paraesophageal Hernia Repair. Thorac. Surg. Clin. 2019; 29 (4): 395-403.

16. Guidelines for Surgical Treatment of Gastroesophageal Reflux Disease (GERD). Society of American Gastrointestinal and Endoscopic Surgeons. 2020.

\section{INFORMATION ABOUT AUTHORS}

Zubarev Petr N. - M. D., D. Sc. (Medicine), Associate Professor, Associate Professor of the General Surgery Department, S. M. Kirov Military Medical Academy of the Russian Defense Ministry, bld. 6, Akademika Lebedeva str., Saint Petersburg, Russia, 194044

Khokhlov Aleksey V. - M. D., D. Sc. (Medicine), Professor, Professor of the General Surgery Department, S. M. Kirov Military Medical Academy of the Russian Defense Ministry, bld. 6, Akademika Lebedeva str., Saint Petersburg, Russia, 194044

Onnitsev Igor E. - M. D., D. Sc. (Medicine), senior lecturer of the General Surgery Department, S. M. Kirov Military Medical Academy of the Russian Defense Ministry, bld. 6, Akademika Lebedeva str., Saint Petersburg, Russia, 194044

Trofimov Vladislav M. - M. D., D. Sc. (Medicine), Professor, Professor of the General Surgery Department, S. M. Kirov Military Medical Academy of the Russian Defense Ministry, bld. 6, Akademika Lebedeva str., Saint Petersburg, Russia, 194044

Belevich Valeriy L. - M. D., D. Sc. (Medicine), senior lecturer of the General Surgery Department, S. M. Kirov Military Medical Academy of the Russian Defense Ministry, bld. 6, Akademika Lebedeva str., Saint Petersburg, Russia, 194044

Muzharovskiy Anton L. - M. D., Ph. D. (Medicine), surgeon of the General Surgery Department, S. M. Kirov Military Medical Academy of the Russian Defense Ministry, bld. 6, Akademika Lebedeva str., Saint Petersburg, Russia, 194044 